Article

\title{
Giant photoresponse in quantized SrRuO monolayer at oxide interfaces
}

Heng-Jui Liu, Jing-Ching Wang, Deok-Yong Cho, Kang-Ting Ho, Jheng-Cyuan

Lin, Bo-Chao Huang, Yue-Wen Fang, Yuan-Min Zhu, Qian Zhan, Lin Xie, Xiao-

Qing Pan, Ya-Ping Chiu, Chun-Gang Duan, Jr-Hau He, and Ying-Hao Chu

ACS Photonics, Just Accepted Manuscript • DOI: 10.1021/acsphotonics.7b01339 • Publication Date (Web): 31 Jan 2018

Downloaded from http://pubs.acs.org on February 6, 2018

\section{Just Accepted}

"Just Accepted" manuscripts have been peer-reviewed and accepted for publication. They are posted online prior to technical editing, formatting for publication and author proofing. The American Chemical Society provides "Just Accepted" as a service to the research community to expedite the dissemination of scientific material as soon as possible after acceptance. "Just Accepted" manuscripts appear in full in PDF format accompanied by an HTML abstract. "Just Accepted" manuscripts have been fully peer reviewed, but should not be considered the official version of record. They are citable by the Digital Object Identifier (DOI®). "Just Accepted" is an optional service offered to authors. Therefore, the "Just Accepted" Web site may not include all articles that will be published in the journal. After a manuscript is technically edited and formatted, it will be removed from the "Just Accepted" Web site and published as an ASAP article. Note that technical editing may introduce minor changes to the manuscript text and/or graphics which could affect content, and all legal disclaimers and ethical guidelines that apply to the journal pertain. ACS cannot be held responsible for errors or consequences arising from the use of information contained in these "Just Accepted" manuscripts. 


\section{Giant photoresponse in quantized $\mathrm{SrRuO}_{3}$ monolayer at oxide} interfaces

Heng-Jui Liu, ${ }^{\dagger}$ Jing-Ching Wang, ${ }^{*}$ Deok-Yong Cho, ${ }^{s}$ Kang-Ting Ho," Jheng-Cyuan

Lin, ${ }^{¥}$ Bo-Chao Huang, ${ }^{¥}$ Yue-Wen Fang, ${ }^{£, \propto}$ Yuan-Min Zhu, ${ }^{\natural, \#}$ Qian Zhan, ${ }^{\natural}$ Lin Xie, ${ }^{\Delta, \$}$

Xiao-Qing Pan, ${ }^{\$, \diamond}$ Ya-Ping Chiu, ${ }^{\sharp, \perp}$ Chun-Gang Duan, ${ }^{£}$ Jr-Hau He, ${ }^{\|}$Ying-Hao

$C h u^{\#, \circ, *}$

${ }^{\dagger}$ Department of Materials Science and Engineering, National Chung Hsing University, Taichung 40227, Taiwan.

Department of Physics, National Sun Yat-sen University, Kaohsiung 80424, Taiwan

${ }^{\S}$ IPIT \& Department of Physics, Chonbuk National University, Jeonju 54896, Republic of Korea.

${ }^{\|}$Computer, Electrical and Mathematical Sciences and Engineering, King Abdullah University of Science and Technology, Thuwal, 23955-6900, Kingdom of Saudi Arabia.

${ }^{¥}$ Institute of Physics, Academia Sinica, Taipei 11529, Taiwan.

${ }^{£}$ Key Laboratory of Polar Materials and Devices, Ministry of Education, East China Normal University, Shanghai 200241, China.

${ }^{p}$ Nanostructures Research Laboratory, Japan Fine Ceramics Center, Nagoya 456-8587, Japan.

IDepartment of Material Physics and Chemistry, University of Science and Technology Beijing, Beijing 100083, China.

"National Center for Electron Microscopy in Beijing, School of Materials Science and Engineering, Tsinghua University, Beijing 100084, China. 
${ }^{\Delta}$ National Laboratory of Solid State Microstructures and College of Engineering and Applied Sciences, Nanjing University, Nanjing, Jiangsu 210093, China

${ }^{\$}$ Department of Chemical Engineering and Materials Science, University of California, Irvine, CA 92697, USA

${ }^{\circ}$ Department of Physics and Astronomy, University of California-Irvine, CA 92697, USA

${ }^{\perp}$ Department of Physics, National Taiwan University, Taipei 10617, Taiwan.

${ }^{\circ}$ Department of Materials Science and Engineering, National Chiao Tung University Hsinchu 30010, Taiwan.

KEYWORDS: $\mathrm{SrRuO}_{3}$ monolayer, Complex oxide heterostructures, Photoresponse, Optoelectronics, interface engineering 


\begin{abstract}
The photoelectric effect in semiconductors is the main mechanism for most modern optoelectronic devices, in which the adequate bandgap plays the key role for acquiring high photoresponse. Among numerous material categories applied in this field, the complex oxides exhibit great possibilities because they present a wide distribution of band gaps for absorbing light with any wavelength. Their physical properties and lattice structures are always strongly coupled and sensitive to light illumination. Moreover, the confinement of dimensionality of the complex oxides in the heterostructures can provide more diversities in designing and modulating the band structures. On the basis of this perspective, we have chosen itinerary ferromagnetic $\mathrm{SrRuO}_{3}$ as the model material, and fabricated it in one-unit-cell thickness in order to open a small band gap for effective utilization of visible light. By inserting this $\mathrm{SrRuO}_{3}$ monolayer at the interface of the well-developed two-dimensional electron gas system $\left(\mathrm{LaAlO}_{3} / \mathrm{SrTiO}_{3}\right)$, the resistance of the monolayer can be further revealed. In addition, a giant enhancement $(>300 \%)$ of photoresponse under illumination of visible light with power density of $500 \mathrm{~mW} / \mathrm{cm}^{2}$ is also observed. Such can be ascribed to the further modulation of band structure of the $\mathrm{SrRuO}_{3}$ monolayer under the illumination, confirmed by cross-section scanning tunneling


microscopy (XSTM). Therefore, this study demonstrates a simple route to design and explore the potential low dimensional oxide materials for future optoelectronic devices. 
Over the past decades, low dimensional materials have attracted tremendous interests in the applications of novel optoelectronics. The confinement of electrons in nanocrystals exhibits high flexibility in the energy-relaxation pathway of photoexcited electron-hole pairs. ${ }^{1}$ Greatly enhanced photocurrent due to the generation of multiple excitons has been observed in zero-dimensional (0D) colloidal quantum-dot, ${ }^{2-4}$ and one-dimensional (1D) carbon nanotube systems. ${ }^{5-6}$ Recently, two-dimensional (2D) materials such as graphene and transition-metal dichalcogenide (TMD) monolayers, also become another fast-growing branch in this research field due to their extraordinary electrical and optical properties. Graphene, as a zero-bandgap material, has an extremely broad absorption spectrum from far-infrared to ultraviolet light. $^{7-8}$ Its linear energy-momentum dispersion allows intriguing mechanisms of electron excitation by absorbing light while being placed in specific designed devices or heterostructures. ${ }^{1,9-12}$ Compared to the graphene-based devices, the TMD monolayers are direct-bandgap semiconductors $(1 \sim 2 \mathrm{eV})$, which possess remarkably stronger photoresponse within the visible light range, ${ }^{13-16}$ and can be complementary to the drawbacks of graphene. These studies clearly reveal that quantum confinement effect and adequate bandgap engineering can lead to superior light-dependent functionalities of the low dimensional materials than their bulk counterparts.

In addition to the low dimensional materials, transition-metal oxides, especially 
the Mott insulators with narrow-bandgap, have been considered as another category of potential optoelectronic materials as well. The theoretical works proposed by Manousakis et al. ${ }^{17-18}$ have predicted that the enhancement of quantum efficiency through photo-induced carrier multiplication process may happen in those Mott insulators within the band gap range of $0.5-1 \mathrm{eV}$. However, in the bulk Mott insulators like $\mathrm{LaVO}_{3}$, the presence of high-density charge-trapping defects and the formation of polarons usually hamper additional multiple electron-hole pairs, leading to low performance of the photo-induced transport behavior. ${ }^{19}$ Therefore, it would be intriguing if we can fabricate the high quality Mott insulator oxides in low dimensionality. On the basis of quantum confinement effect, while the size of materials is reduced to nanoscale, the energy bands become discrete and the band gap becomes wider. Hence, to explore the potential material systems, the transition-metal oxides with very small bandgap or no bandgap have been conceptually preferred. Previous reports have demonstrated that the metallic oxides, such as $\mathrm{SrRuO}_{3}$ $(\mathrm{SRO})^{20-22}$ or $\mathrm{LaNiO}_{3}(\mathrm{LNO}){ }^{23}$ present a metal-to-insulator transition when the thickness decreases below several unit cells, which offers the possibilities of meeting the criteria for high performance in optoelectronic devices.

In this work, the SRO monolayer has been chosen for investigating the photoresponse because it presents wide absorption spectrum in its bulk form. ${ }^{24}$ By 
using the merits of epitaxial oxide heterostructures, where versatile electronic reconstructions and electron-phonon interactions can be procured at the interfaces, the electronic state of the SRO monolayer can be further modulated. To achieve this purpose, a specifically designed sample architecture, similar to the cases with an inserted one-unit-cell manganite between $\mathrm{LaAlO}_{3} \quad(\mathrm{LAO}) / \mathrm{SrTiO}_{3} \quad$ (STO) heterostructures, ${ }^{25-26}$ has been adopted as illustrated in the schematic of Figure 1a. $\mathrm{LAO} / \mathrm{STO}$ is a famous system that possesses $2 \mathrm{D}$ electron gas (2DEG) behavior, which results from structural and electronic reconstruction at its interface. ${ }^{27-30}$ Therefore, the LAO capping layer is also expected to cause certain variation on the sandwiched SRO monolayer. Further verifications have been brought by electrical transport measurement, X-ray absorption spectroscopy (XAS) and cross-section scanning tunneling microscopy (XSTM). Excitingly, a large enhancement of photocurrent relative to dark-current $(\sim 325 \%)$ is obtained under the illumination with power density of $500 \mathrm{~mW} / \mathrm{cm}^{2}$, which confirms the feasibility of creating and modulating the 2D oxide systems for designing new optoelectronic devices.

\section{Results and discussion}

The high-quality samples for investigation were fabricated using pulsed laser deposition (PLD) equipped with reflection high-energy electron diffraction (RHEED). To accurately control the thickness of the SRO monolayer in one unit cell, 
$\mathrm{TiO}_{2}$-terminated (001) STO single crystal substrates with atomically flat surface were required before the growth. The preparation uses the common acid treatment method proposed by Kawasaki, et al. ${ }^{31}$ Under the optimized growth conditions (please see experimental section), the deposition of the SRO monolayer and the LAO capping layer can follow the layer-by-layer epitaxy mechanism. As shown in Figure 1b, the SRO monolayer was obtained by stopping the deposition after finishing one clear intensity oscillation in RHEED curve. The subsequent LAO capping layer was also precisely controlled in 6 unit cells from the RHEED monitoring. Here this sample is designated as $\mathrm{LAO} / \mathrm{SRO} / \mathrm{STO}$. The morphology of the sample probed by atomic force microscopy (AFM) as the inset in Figure $1 \mathrm{~b}$ reveals an atomically smooth surface with regular terraces and a step height of $\sim 4 \AA$, confirming the layer-by-layer epitaxial growth. It is worth noting that the SRO naturally prefers to end up with $\mathrm{SrO}$ termination while grown on the $\mathrm{TiO}_{2}$ terminated STO substrate, which implies that the $\mathrm{RuO}_{2}$ plane is sandwiched between two SrO planes in this case. ${ }^{32}$ Figure 1c exhibits the typical atomic-resolution Z-contrast scanning transmission electron microscopy (STEM) image along [010] direction of the STO substrate. According to the principle in the Z-contrast image, the contrast between two A-site elements, La and $\mathrm{Sr}$, can be easily distinguished due to their large difference of atomic numbers $\left(Z_{\mathrm{La}}=57\right.$ and $Z_{\mathrm{Sr}}=38$, respectively). Thus, the La atoms exhibit brighter contrast than 
the $\mathrm{Sr}$ atoms. The resembling phenomenon can be observed at three B-site elements $\left(Z_{\mathrm{Ti}}=22\right.$ and $\mathrm{Z}_{\mathrm{Ru}}=44$, and $\left.\mathrm{Z}_{\mathrm{Al}}=13\right)$ as well. By identifying these atoms in the Z-contrast images, the SRO monolayer has been confirmed to be coherently connected to the neighboring STO substrate and the LAO capping layer without detectable linear defects, presenting a high quality epitaxial growth of the designed heterostructure.

We have than examined the electronic transport behavior of this SRO monolayer. Similar to the studies presented in several previous studies ${ }^{20-22}$, the resistance of this SRO monolayer presents an expectable semiconducting behavior (Figure 2a) as well. To understand the transport mechanism dominating in the SRO monolayer (Figure 2b), we firstly adopt the thermal activation model to fit the curve of temperature dependent resistance using Arrhenius equation:

$$
R(T)=R_{0} \exp \left(E_{A} / k_{B} T\right)
$$

where $T$ is the absolute temperature, $R_{0}$ is a prefactor that represents the value of resistance at infinite temperature, $E_{\mathrm{A}}$ is the activation energy and $k_{\mathrm{B}}$ is the Boltzmann's constant. The conduction mechanism of this model depends on the thermally excited electrons from the valence band or the impurity levels to the conduction band by overcoming the bandgap. The linear fit of $\ln (R(T))$ versus $1 / T$ shows a good agreement with the experimental data in the high temperature range of 
$230 \mathrm{~K}-300 \mathrm{~K}$, in which the thermal activation energy $E_{A}$ of $\sim 170 \mathrm{meV}$ is extracted according to the slope. However, the model quickly fails below $230 \mathrm{~K}$, implying that this conduction type is insufficient to be used to interpret the transport behavior of the SRO monolayer at low temperature. Therefore, the Mott variable range hopping (VRH), widely used for describing the transport behavior in many strongly correlated electron systems, has been also adopted. The temperature dependence of resistance in the Mott VRH expression can be simplified as the following equation:

$$
R(T)=R_{0} \exp \left(T_{0} / T\right)^{1 / \alpha}
$$

where prefactor $R_{0}$ is a constant derived from the electron-phonon interaction, $T_{0}$ represents the degree of charge carrier localization, determined by the density of localized states at the Fermi level $\left(\mathrm{N}\left(\mathrm{E}_{\mathrm{F}}\right)\right)$ and the localization length $(\xi)$ : $T_{0} \approx 27 /\left(\pi k_{\mathrm{B}} \mathrm{N}\left(\mathrm{E}_{\mathrm{F}}\right) \xi^{2}\right) \cdot{ }^{33-35}$ Moreover, the energy for electron hopping is also a function of temperature, and $\alpha$ is the dimension of system. For a 3D or $2 \mathrm{D}$ system, $\alpha$ corresponds to 4 or 3 , respectively. According to this model, the electrons near the Fermi level are localized with random potential fluctuations, and the conduction principally relies on long-distance hopping of these electrons at these localized states by receiving energy from phonons. Therefore, the hopping energy $(\Delta E)$ can be expressed as the equation (3):

$$
\Delta E=\frac{1}{4} k_{B} T\left(T_{0} / T\right)^{1 / \alpha}
$$


This equation describes that when temperature decreases, the energy for electron hopping increases. Here a better fit at wider temperature range from $150 \mathrm{~K}$ to $300 \mathrm{~K}$ can be obtained when the model of 2D Mott variable range hopping (VRH) is used:

$$
R(T)=R_{0} \exp \left(T_{0} / T\right)^{1 / 3}
$$

It implies that the carriers in this inserted SRO monolayer are strongly localized and confined between LAO and STO, and hence 2D hopping behavior is reasonably responsible for the transport nature. However, we still observe a deviation of the experimental data from the theoretical fitting curves below $150 \mathrm{~K}$. This critical temperature is very close to the magnetic phase transition of bulk SRO. Although there is still no evidence to support the existence of phase transition in this SRO monolayer, it is believed that increasing magnetic disorder with decreasing temperature in the SRO monolayer could happen and result in the change of the electrical transport behavior. As a result, merely the 2D Mott VRH mode cannot perfectly fit the data as well, and the conduction may involve more other mechanisms at low temperature. From the analysis of the transport data, excitingly, both models reveal the similar results that a gap has now been created in the energy band of this SRO monolayer, which satisfies the first criterion for high efficient optoelectronics. It is noteworthy that no study has experimentally revealed electrical transport behavior of the SRO monolayer thus far. The thinnest SRO film with the detectable 
resistance is 2 unit cells, reported by Chang, et al. ${ }^{21}$ Therefore, in order to realize how the LAO capping layer assists this SRO monolayer to acquire the measurable resistance (even though it is still very high), we performed the x-ray absorption spectroscopy (XAS) to reveal its electronic structure. Here we prepared other two samples, the SRO monolayer capped with 6-unit-cell STO layer and 100-unit-cell SRO film capped with 6-unit-cell LAO layer (termed as STO/SRO/STO and $\mathrm{LAO} / 100$ u.c. SRO/STO, respectively), and $\mathrm{SRO}$ and $\mathrm{RuO}_{2}$ powders for comparison with $\mathrm{LAO} / \mathrm{SRO} / \mathrm{STO}$ sample. STO/SRO/STO is a very important control sample, in which the STO capping layer can not only keep the structural completeness of the SRO monolayer from the symmetry breaking effect at exposed surface, but also provide another kind of electrostatic boundary condition to the SRO monolayer compared to LAO/SRO/STO sample. ${ }^{26}$ We have also found that the resistance of $\mathrm{STO} / \mathrm{SRO} / \mathrm{STO}$ is extremely high and exceeds the measurement limit of our instrument. It is consistent with the present studies mentioned above that the resistance of the SRO monolayer is hardly being detected. The corresponding evidence will be given in the latter photoresponse measurement.

Figure $3 \mathrm{a}$ indicates the possible chemical influence on the local environment of $\mathrm{Ru}$ ions from the neighboring capping layers in the XAS spectra across the $\mathrm{Ru}$ $L_{3}$-edge ( $\mathrm{h} v \sim 2840 \mathrm{eV}$ for $\mathrm{Ru}^{4+}$ ) with the light polarization perpendicular to the 
surface normal $\left(I_{E / / a b}\right)$. All samples exhibit nearly the same position of the absorption peak, implying no obvious valence change for Ru ions for these samples. Recall that the deposited SRO monolayer naturally prefers to transform B-site termination to A-site termination. ${ }^{32}$ By following the concept from the LAO thin film grown on the SrO-terminated STO system, this top SrO plane should be oxygen-deficient and create a positive built-in electric field toward surface. ${ }^{36}$ Simultaneously, it also helps to hinder the electrons transferring to the SRO monolayers, and thus maintains the valence of SRO. The retention of $\mathrm{Ru}^{4+}$ can be further confirmed by the XAS spectra across the $\mathrm{O} K$-edge, as shown in Figure $3 \mathrm{~b}$ and Supplementary Fig. S1, where the Ru $4 d-\mathrm{O} 2 p(\sim 529.04 \mathrm{eV})$ and Ti $3 d-\mathrm{O} 2 p(\sim 530.86 \mathrm{eV})$ hybridizations are revealed. Although the peak for the $\mathrm{Ru} 4 d$ state in the SRO monolayer is very broad, its peak position still locates at nearly the same energy with that of the bulk SRO film, suggesting that no obvious oxygen deficiency occurs at this $\mathrm{RuO}_{2}$ plane. Similar inference can be also obtained from the Ti $3 d$ state, which has no obvious peak shift between the samples of the SRO monolayer and the 100-unit-cell SRO film. Because the Ti $3 d$ state only reflects the oxidation for the STO substrate, the unchanged valence state of $\mathrm{Ti}$ ions supports that charge transfer can be effectively protected at the top SrO plane. Moreover, the spectra of STO/SRO/STO and LAO/SRO/STO both exhibit a double-peak feature, where the most intense peaks $\left(e_{g}\right.$ levels $)$ and pre-peaks 
( $t_{2 g}$ levels) are highlighted by the dashed lines in the figure. Compared to the reference samples, the line shape of STO/SRO/STO is closer to that of the SRO powder, whereas the line shape of LAO/SRO/STO is resembling to that of the $\mathrm{RuO}_{2}$ powder. While the thickness of this inserted SRO layer is increased to 100 unit cells, its line shape becomes the same with that of the SRO powder again, implying that the 100-unit-cell SRO thin film maintains the bulk properties and the effect of the LAO capping layer can be ignored. The $t_{2 g}-e_{g}$ crystal field splitting in the SRO monolayer is realized from the breaking of degeneracies of electron orbital states in $\mathrm{Ru}$ ions due to the produced electrostatic field while being placed in the octahedrons. Further orbital splitting of the $e_{g}$ and $t_{2 g}$ states caused by the Jahn-Teller effect appears when a strain or deformation is introduced into these octahedrons. The higher the intensity ratio of main peak to pre-peak $\left(e_{g} / t_{2 g}\right)$ is, the less distortion the Ru octahedron has. ${ }^{37}$ Therefore, in this work, the SRO monolayer possesses less lattice distortion while being covered by the LAO than the STO thin film. Such can be attributed to the relaxation by the conceivable presence of point defects like oxygen vacancies produced in the top $\mathrm{SrO}$ layer during high vacuum deposition process for LAO. The possible mechanism for how the lattice variation leads to the modulation of resistance has then been described in the schematic of Figure 3c. In bulk SRO, the Fermi level is located within the valence band of the $\mathrm{Ru} t_{2 g}$ orbital that usually exhibits a low spin 
states $\left(t_{2 g}^{4} e_{g}^{0}\right)$ and results in the corresponding metallic behavior. When its dimensionality is reduced to the monolayer, we supposedly observe a band gap that will be confirmed in the following measurement of cross-sectional scanning tunneling microscopy and spectroscopy (XSTM/S). Hence, the Fermi level has then been located at gap between the $\mathrm{Ru} t_{2 g}$ and $e_{g}$ orbitals. With relaxing lattice distortion of SRO, smaller band gap between $t_{2 g}$ and $e_{g}$ due to crystal field splitting is anticipated and leads to lower resistance observed in LAO/SRO/STO. From previous studies ${ }^{25-26}$, we have realized that charge transfer is originally responsible for control of transport behavior of the manganite monolayers. Nevertheless, no variation of $\mathrm{Ru}$ valence could be seen in the case with either the STO or the LAO capping layer, suggesting that modulation of lattice distortion should dominate the transport behavior of the SRO monolayer rather than charge transfer effect.

The band gap variation of the SRO monolayers in the cases with different capping layers can be further supported by the density-functional theory (DFT) calculations as shown in Figure 4. To investigate the properties of the SRO monolayer at the interfaces, we constructed two cases of slab models, each of which has two symmetric interfaces. The case 1 is $(\mathrm{STO})_{6}-(\mathrm{SRO})_{1.5}-(\mathrm{STO})_{5.5}-(\mathrm{SRO})_{1.5}-(\mathrm{STO})_{6}$, denoted as case $1-\mathrm{L} 6$. The case 2 is $(\mathrm{LAO})_{\mathrm{n}}-(\mathrm{SRO})_{1.5}-(\mathrm{STO})_{5.5}-(\mathrm{SRO})_{1.5}-(\mathrm{LAO})_{\mathrm{n}}$, in which $\mathrm{n}=1$ for case $2-\mathrm{L} 1$ and $\mathrm{n}=2$ for case $2-\mathrm{L} 2$, respectively. Figure 4 shows 
the layer projected density of states in the SRO monolayer of case $1-$ L6, case $2-\mathrm{L} 1$, and case $2-\mathrm{L} 2$. A band gap of around $0.4 \mathrm{eV}$ is displayed in case $1-\mathrm{L} 6$, which is in good agreement with Li et al.'s recent predictions of the undoped SRO monolayer. ${ }^{38}$ On the other hand, the band gap of the SRO monolayer in case $2-\mathrm{L} 1$ is estimated to be $0.15 \mathrm{eV}$, which is obviously narrowed compared to that in case $1-$ L6. This narrowing effect is much more significant in case $2-\mathrm{L} 2$, where the SRO monolayer shows spin-polarized conducting state, as indicated by the corresponding layer projected density of states. When the unit-cell number of the LAO capping layer increases above 2 unit cells, all simulated results are almost the same with case $2-$ L2. Therefore, the comparison among these three cases is enough. In experiment, although the resistance in $\mathrm{LAO} / \mathrm{SRO} / \mathrm{STO}$ is still high with presenting a semiconducting feature, we can find that it is relatively smaller than that in STO/SRO/STO. The difference between theory and experiment data may be originated from some non-perfect condition in samples such as temperature effect because all theoretic results are constructed on the calculation adopting absolute $0 \mathrm{~K}$, whereas the experimental results can only be obtained at above $60 \mathrm{~K}$. However, the tendency is similar for both theory and experiment, indicating that the band gap of the SRO monolayer with the LAO capping layer is intrinsically smaller than that with the STO capping layer. 
The photoresponse of the SRO monolayer was then conducted by a simple e-beam lithography pattern with four square electrodes connecting to the interface. As shown in Figure 5a, the light illumination was focused on the center area between the electrodes. The change of current under the illumination is defined as photocurrent: $\Delta \mathrm{I}$ $=I_{\text {light }}-I_{\text {dark }}$, where $I_{\text {dark }}$ is the dark current before illumination and $I_{\text {light }}$ is the obtained current under the illumination. The ratio of $\Delta \mathrm{I} / \mathrm{I}_{\text {dark }}$ is then defined as photoresponse. Figure $5 b$ is the current-voltage (I-V) curves (in logarithmic scale) of both $\mathrm{LAO} / \mathrm{SRO} / \mathrm{STO}$ and $\mathrm{STO} / \mathrm{SRO} / \mathrm{STO}$ samples measured in the dark and under illumination of ultraviolet light source with $\lambda=350 \mathrm{~nm}$ and power density of 0.5 $\mathrm{mW} / \mathrm{cm}^{2}$. The measured dark current of $\mathrm{LAO} / \mathrm{SRO} / \mathrm{STO}$ is around one order of magnitude larger than that of STO/SRO/STO. In addition, STO/SRO/STO also presents an unstable dark current at applied voltage range below $2.5 \mathrm{~V}$ due to a very high resistance that is close to the instrumental measurement limit, which is $\sim 1 \mathrm{pA}$. If the practical current obtained from sample is below this value, it is always presented as noisy signals even though we can still observe the "fake" current from the instrument. It implies that the practical current of STO/SRO/STO at an applied field below $\sim 2.5 \mathrm{~V}$ is far smaller than the limit, and the observed current floats with very large fluctuations. On the other hand, the current of LAO/SRO/STO rapidly increases with applied voltage, where the measured current is always above $1 \mathrm{pA}$ that only 
requires the field larger than $0.15 \mathrm{~V}$. Therefore, such abnormal fluctuations are hardly observed in the case of LAO/SRO/STO. This result confirms that the electronic transport behavior of the SRO monolayer can be effectively improved by the LAO capping layer, which is consistent with the conclusions of XAS and theory prediction. When both samples are illuminated, their currents exhibit a large increment with nearly the same magnitude (two orders of magnitude) at all applied voltage range, implying that such an enhancement is indeed caused by this inserted SRO monolayer. Basically, this phenomenon cannot be observed if we replace the SRO monolayer by highly conductive interlayer or interface, i.e., LAO/100 u.c. SRO/STO or 2DEG LAO/STO, respectively. From Supplementary Fig. S2, we have revealed that either $\mathrm{LAO} / 100$ u.c. SRO/STO or 2DEG LAO/STO has undistinguishable variation of current under the same illumination condition, which is in accordance with the expectation that the metallic characteristic cannot afford large photoresponse. Similar results for the $50 \mathrm{~nm}$ SRO thin film with a very small photoresistivity of $\sim 0.2 \%$ to $2 \%$ can be obtained in our previous studies. ${ }^{39-40}$ These observations support that only the opened small bandgap in the SRO monolayer can originate large photoresponse in this kind of oxide heterstructures. Therefore, further exploration of the photocurrent in the semiconducting SRO monolayer at the interface between the LAO layer and the STO substrate becomes very attractive. Figure $5 \mathrm{c}$ shows the photocurrent of the SRO 
monolayer at three typical wavelengths (UV (405 nm), green $(520 \mathrm{~nm})$, and red (642 $\mathrm{nm})$ ). Before the illumination, $\mathrm{I}_{\mathrm{dark}}$ of the SRO monolayer is $\sim 1.8 \times 10^{-10}$ A operated at $5 \mathrm{~V}$. Under the illumination, the power densities of all light sources were kept at 3.6 $\mathrm{mW} / \mathrm{cm}^{2}$ for comparison. Obviously, as the photon energy of light decreases, the photocurrent becomes smaller, which can be attributed to the weaker ability to generate photo-excited carriers under the light with smaller photon energy. For more practical application, we then chose the green light source (in the visible light range) to perform the power dependent measurements. At this wavelength (Figure 5d), the photocurrent increases with power density. A large photoresponse of $\sim 325 \%$ can even be obtained when the power density achieves to $500 \mathrm{~mW} / \mathrm{cm}^{2}$. It clearly reveals that this SRO monolayer has strong light-electron interaction to greatly improve its intrinsically poor transport behavior.

To explore the reason of large photoresponse of the SRO monolayer directly, the XSTM/S operated in an ultrahigh vacuum (UHV) chamber with a light source (laser diode, $\lambda=405 \mathrm{~nm}$, power intensity $=3 \mathrm{~mW} / \mathrm{cm}^{2}$ ) was used. This technique provides the local topography and the corresponding electronic information under illumination, which can reveal the photo-induced change in the band alignments of the SRO monolayer at the atomic spatial resolution. In this measurement, the $0.5 \mathrm{wt} . \% \mathrm{Nb}$ doped STO substrates (Nb-STO) substrate and the protecting thick film of amorphous SRO 
(a-SRO) were required to avoid crashing the tip during the scan. Therefore, the 6-unit-cell STO film was deposited on the Nb:STO substrate at first to mimic original environment of LAO/SRO/STO sample. Afterward the SRO monolayer and the LAO capping layer were sequentially grown on STO. Finally, the surface was covered by a conducting a-SRO film with the thickness greater than $500 \mathrm{~nm}$ for protection. After finishing the growth, the sample was transferred in an ultra-high vacuum (UHV) chamber to proceed the following cleavage and probe of the sample. ${ }^{41}$ The measurement method is schematized in Figure 6a. Before the illumination, Figure $6 \mathrm{~b}$ displays a typically cross-sectional STM topographic image at a sample bias of $+2.0 \mathrm{~V}$. On the basis of the different electronic properties for STO, SRO and LAO, each region can be clearly distinguished. The Nb:STO substrate reveals atomically flat terraces, and height profile changes drastically at the interface between the Nb:STO and the STO buffer layer as indicated by a black arrow in Figure 6b. Moreover, a brighter region about $0.4 \mathrm{~nm}$ between the STO buffer layer and LAO, which can be identified as the SRO monolayer. The detailed information for the comparison of the photo-induced changes of the SRO monolayer can be unveiled by the spectroscopic $\mathrm{dI} / \mathrm{dV}$ results shown in Figure. 6c to 6h. Excitingly, a spectacularly electronic change at the SRO monolayer has been indeed observed, in which the interface exhibits a brighter contrast while being illuminated exhibited in Figures $6 \mathrm{c}$ and $6 \mathrm{~d}$. More clear variation of the band 
alignments across the SRO monolayer for these two conditions is then carried out by the quantitative analysis of the normalized $\mathrm{dI} / \mathrm{dV}$ spectroscopic results, as shown in Figures. 6e and $6 \mathrm{f}$, respectively. ${ }^{42}$ By extracting the intensity profile at the SRO monolayer, we are able to observe its energy gap directly. Before the illumination, the SRO monolayer possesses a small energy gap around $0.8 \mathrm{eV}$ (Figure 6g), supporting the presence of semiconducting behavior. When the sample is irradiated, the energy gap of the SRO monolayer is reduced to $0.6 \mathrm{eV}$ and the corresponding density of states (DOS) increases as well (Figure 6h). Such a reduction of energy gap under illumination is possibly from the structural variation of the SRO monolayer due to the photostriction effect and photo-excited electrons accumulated at the interface. ${ }^{39-40}$ Hence, the electronic conductivity of the SRO monolayer can be greatly enhanced under illumination.

\section{Conclusions}

In this work, we have demonstrated the fabrication of the complex oxide monolayers inserted in specially designed oxide heterostructures through the layer-by-layer growth mechanism and their potential in the optoelectronic devices. On the basic concept of the quantum confinement effect, we can start with a complex oxide, like SRO adopted in this study that originally possesses metallic characteristic in its bulk counterpart, and then force it to open a small bandgap and become semiconducting by limiting the 
thickness in one unit cell. Compared to the van-der-Waal-based 2D materials, such kinds of complex oxide monolayers can exhibit a superior advantage that their electronic structures and physical properties are more easily modulated through the strain engineering. For example, the band gap of the SRO monolayers can be adjusted by altering different capping layer, STO or LAO, through their strong orbital-lattice coupling. The electronic transport behavior of the SRO monolayer is more improved by capping LAO than STO, which is due to the asymmetric-electrostatic-boundaries-induced lattice relaxation. As a result, we are able to first reveal the resistance of the SRO monolayer that has never been reported. Here we also examine the photoresponse of this SRO monolayer, where a considerably large enhancement under the illumination of visible light range is observed. From real-time observation of XSTM/S measurement, we have realized that this enhancement can be ascribed to the further reduction of energy gap during the illumination. Therefore, this study has offered a new perspective to explore the promising $2 \mathrm{D}$ oxide monoalyers for optoelectronics.

\section{Materials and Methods}

Sample Preparation: The samples were fabricated on $\mathrm{TiO}_{2}$-terminated STO (100) substrates using PLD. During the deposition, the stoichiometric targets of SRO, LAO, and STO were used, and the growth of monolayer and capping layers were precisely 
controlled in layer by layer monitored by a RHEED. The substrate temperature and the oxygen pressure were maintained at $700{ }^{\circ} \mathrm{C}$ and 100 mTorr, respectively, for the growth of SRO and STO. However, to avoid the possible 3D growth of the LAO capping layer, the pressure decreased to $5 \times 10^{-5}$ Torr to ensure the layer-by-layer growth. ${ }^{43}$ After the deposition, all the films were post-annealed at the oxygen pressure of 300 Torr for $30 \mathrm{~min}$, and then cooled down to room temperature.

\author{
Structure Characterization by STEM Z-contrast imaging: Structure \\ characterization by Z-contrast imaging was carried out on a JEM-ARM200F \\ microscope, equipped with a cold field emission electron gun, Cs-probe corrector and \\ Cs-image corrector with a minimum probe of about $0.078 \mathrm{~nm}$ in diameter. The probe \\ convergence angle and the detection angle used for imaging were $25 \mathrm{mrad}$ and $92-$ \\ $228 \mathrm{mrad}$, respectively.
}

X-ray Absorption spectroscopy: The Ru L-edge XAS was performed at the 16A1 beamline in Taiwan Light Source. The fluorescence from SRO was collected using a Lytle detector. The O K-edge XAS was performed at the 2A beamline in Pohang Light Source in total electron yield mode. The direction of the incident X-rays was kept perpendicular to the sample planes so that the polarization vector would reside in the SRO plane. 
Cross-Section Scanning Tunneling Microscopy and Spectroscopy: Before the STM measurements, amorphous SRO capping layers $(>500 \mathrm{~nm})$ were deposited on top of all samples to prevent tip crash during the measurements. These samples were cleaved in situ in an UHV chamber whose base pressure was approximately $5 \times 10^{-11}$ Torr. The cross-sectional STM topography images were obtained using constant current mode at $+2.0 \mathrm{~V}$. The corresponding atomically resolved spectroscopic results under illumination and dark conditions were recorded with the first derivative of tunneling current over tip-sample voltage (or differential conductivity), dI/dV. The spatial resolution is around $0.4 \mathrm{~nm}$ at interfaces. In order to reduce the thermal influence in our STS measurements under light illumination, we used the electronic controlled system. The thermal influence could be reduced by intermittently modulating the light output.

DFT calculation: We used first-principles calculation within the framework of density-functional theory to investigate the electronic states of the SRO monolayer. These calculations were performed using the Vienna ab initio simulation package (VASP) ${ }^{44-45}$ in which the exchange-correlation potential is treated in Perdew-BurkeErnzerhof (PBE). Because the on-site electron-electron interactions are strong in the localized $d$ orbitals for $\mathrm{Ru}$, the Hubbard $U$ parameters, i.e., $U=2.5$ and $J=0.4 \mathrm{eV}$ were applied to $\mathrm{Ru} d$ orbitals. These parameters have been extensively used to 
investigate the electronic and magnetic properties of SRO-based interfaces. ${ }^{46}$ The energy cutoff of $500 \mathrm{eV}$ was used for the plane wave expansion of the projector augmented wave and $5 \times 5 \times 1$ Monkhorst-Pack grid is sampled for k-points in the self-consistent calculations.

\section{Electric transport Measurements:}

In order to conduct the transport measurements, four square metallic ( $\mathrm{Au} / \mathrm{Ti})$ electrodes $\left(0.3 \mathrm{~mm}^{2}\right.$ size $)$ connected to the sandwiched SRO monolayer were thermally evaporated on the samples using e-beam lithography. Then gold wires were manually bonded to the electrodes using silver paste. The typical transport measurement was performed on Cryogen-free $4 \mathrm{~K}$ cryogenic probe station (Lake Shore Model CRX-4K) with high resistance meter (Keithley Model 6517A) for the samples. The resistance was obtained under the DC electric bias $1 \mathrm{~V}$.

\section{Photoresponse measurement:}

Photoresponse of the samples was measured by a semiconductor characterization system (Keithley 4200-SCS with DC preamplifier). During the photoresponse measurements, the samples were illuminated by four kinds of single mode laser diodes: 350, 405, 520, and $642 \mathrm{~nm}$, respectively (Thorlabs modulated by the ITC4001 driver). The light intensity was measured by a power meter. 
ASSOCIATED CONTENT

\section{Supporting Information}

The Supporting Information is available free of charge on the ACS Publications website at DOI:

Fitting results of samples across the XAS spectra of $\mathrm{O} K$ edge; Photo-dependent I-V curves for the samples of STO/SRO/STO, LAO/SRO/STO, LAO/100 u.c. SRO/STO, and common 2DEG system.

\section{- AUTHOR INFORMATION}

\section{Corresponding Author}

*E-mail: yhc@nctu.edu.tw (Y.H.C.).

\section{ORCID}

Ying-Hao Chu: 0000-0002-3435-9084

Heng-Jui Liu: 0000-0002-0745-9370

\section{Author contributions}

H.J.L. and J.C.W. contributed equally.

\section{Notes}

The authors declare no competing financial interest. 


\section{References:}

(1) Gabor, N. M.; Song, J. C. W.; Ma, Q.; Nair, N. L.; Taychatanapat, T.; Watanabe, K.; Taniguchi, T.; Levitov, L. S.; Jarillo-Herrero, P. Hot CarrierAssisted Intrinsic Photoresponse in Graphene. Science 2011, 334, 648-652.

(2) Sukhovatkin, V.; Hinds, S.; Brzozowski, L.; Sargent, E. H. Colloidal Quantum-Dot Photodetectors Exploiting Multiexciton Generation. Science 2009, 324, 1542-1544.

(3) Binks, D. J. Multiple exciton generation in nanocrystal quantum dots controversy, current status and future prospects. Phys. Chem. Chem. Phys. 2011, 13, 12693-12704.

(4) Rabani, E.; Baer, R. Theory of multiexciton generation in semiconductor nanocrystals. Chem. Phys. Lett. 2010, 496, 227-235.

(5) Gabor, N. M.; Zhong, Z.; Bosnick, K.; Park, J.; McEuen, P. L. Extremely Efficient Multiple Electron-Hole Pair Generation in Carbon Nanotube Photodiodes. Science 2009, 325, 1367-1371.

(6) Wang, S.; Khafizov, M.; Tu, X.; Zheng, M.; Krauss, T. D. Multiple Exciton Generation in Single-Walled Carbon Nanotubes. Nano Lett. 2010, 10, 2381-2386.

(7) Bonaccorso, F.; Sun, Z.; Hasan, T.; Ferrari, A. C. Graphene photonics and optoelectronics. Nat. Photon. 2010, 4, 611-622.

(8) Zhang, B. Y.; Liu, T.; Meng, B.; Li, X.; Liang, G.; Hu, X.; Wang, Q. J. Broadband high photoresponse from pure monolayer graphene photodetector. Nat. Commun. 2013, 4, 1811.

(9) Mueller, T.; Xia, F.; Avouris, P. Graphene photodetectors for high-speed optical communications. Nat. Photon. 2010, 4, 297-301.

(10) Liu, M.; Yin, X.; Ulin-Avila, E.; Geng, B.; Zentgraf, T.; Ju, L.; Wang, F.; Zhang, X. A graphene-based broadband optical modulator. Nature 2011, 474, 64-67.

(11) Winzer, T.; Knorr, A.; Malic, E. Carrier Multiplication in Graphene. Nano Lett. 2010, 10, 4839-4843.

(12) Xia, F.; Mueller, T.; Lin, Y.-m.; Valdes-Garcia, A.; Avouris, P. Ultrafast graphene photodetector. Nat. Nanotech. 2009, 4, 839-843.

(13) Sun, Z.; Martinez, A.; Wang, F. Optical modulators with 2D layered materials. Nat. Photon. 2016, 10, 227-238.

(14) Lopez-Sanchez, O.; Lembke, D.; Kayci, M.; Radenovic, A.; Kis, A. Ultrasensitive photodetectors based on monolayer $\mathrm{MoS}_{2}$. Nat. Nanotech. 2013, $8,497-501$. 
(15) Buscema, M.; Barkelid, M.; Zwiller, V.; van der Zant, H. S. J.; Steele, G. A.; Castellanos-Gomez, A. Large and Tunable Photothermoelectric Effect in Single-Layer $\mathrm{MoS}_{2}$. Nano Lett. 2013, 13, 358-363.

(16) Zhang, W.; Chuu, C.-P.; Huang, J.-K.; Chen, C.-H.; Tsai, M.-L.; Chang, Y.-H.; Liang, C.-T.; Chen, Y.-Z.; Chueh, Y.-L.; He, J.-H.; Chou, M.-Y.; Li, L.-J. Ultrahigh-Gain Photodetectors Based on Atomically Thin Graphene- $\mathrm{MoS}_{2}$ Heterostructures. Sci. Rep. 2014, 4, 3826.

(17) Manousakis, E. Photovoltaic effect for narrow-gap Mott insulators. Phys. Rev. B 2010, 82, 125109.

(18) Coulter, J. E.; Manousakis, E.; Gali, A. Optoelectronic excitations and photovoltaic effect in strongly correlated materials. Phys. Rev. B 2014, 90, 165142 .

(19) Wang, L.; Li, Y.; Bera, A.; Ma, C.; Jin, F.; Yuan, K.; Yin, W.; David, A.; Chen, W.; Wu, W.; Prellier, W.; Wei, S.; Wu, T. Device Performance of the Mott Insulator $\mathrm{LaVO}_{3}$ as a Photovoltaic Material. Phys. Rev. Appl. 2015, 3, 064015.

(20) Toyota, D.; Ohkubo, I.; Kumigashira, H.; Oshima, M.; Ohnishi, T.; Lippmaa, M.; Takizawa, M.; Fujimori, A.; Ono, K.; Kawasaki, M.; Koinuma, H. Thickness-dependent electronic structure of ultrathin $\mathrm{SrRuO}_{3}$ films studied by in situ photoemission spectroscopy. Appl. Phys. Lett. 2005, 87, 162508.

(21) Chang, Y. J.; Kim, C. H.; Phark, S. H.; Kim, Y. S.; Yu, J.; Noh, T. W. Fundamental Thickness Limit of Itinerant Ferromagnetic $\mathrm{SrRuO}_{3}$ Thin Films. Phys. Rev. Lett. 2009, 103, 057201.

(22) Shen, X.; Qiu, X.; Su, D.; Zhou, S.; Li, A.; Wu, D. Thickness-dependent metal-insulator transition in epitaxial $\mathrm{SrRuO}_{3}$ ultrathin films. J. Appl. Phys. 2015, 117, 015307.

(23) King, P. D. C.; Wei, H. I.; Nie, Y. F.; Uchida, M.; Adamo, C.; Zhu, S.; He, X.; Bozovic, I.; Schlom, D. G.; Shen, K. M. Atomic-scale control of competing electronic phases in ultrathin $\mathrm{LaNiO}_{3}$. Nat. Nanotech. 2014, 9, 443-447.

(24) Lee, S.; Apgar, B. A.; Martin, L. W. Strong Visible-Light Absorption and Hot-Carrier Injection in $\mathrm{TiO}_{2} / \mathrm{SrRuO}_{3}$ Heterostructures. Adv. Energy Mater. 2013, 3, 1084-1090.

(25) Chen, Y. Z.; Trier, F.; Wijnands, T.; Green, R. J.; Gauquelin, N.; Egoavil, R.; Christensen, D. V.; Koster, G.; Huijben, M.; Bovet, N.; Macke, S.; He, F.; Sutarto, R.; Andersen, N. H.; Sulpizio, J. A.; Honig, M.; Prawiroatmodjo, G. E. D. K.; Jespersen, T. S.; Linderoth, S.; Ilani, S.; Verbeeck, J.; Van Tendeloo, G.; Rijnders, G.; Sawatzky, G. A.; Pryds, N. Extreme mobility enhancement of two-dimensional electron gases at oxide interfaces by charge-transfer-induced modulation doping. Nat. Mater. 2015, 14, 801-806. 
(26) Liu, H.-J.; Lin, J.-C.; Fang, Y.-W.; Wang, J.-C.; Huang, B.-C.; Gao, X.; Huang, R.; Dean, P. R.; Hatton, P. D.; Chin, Y.-Y.; Lin, H.-J.; Chen, C.-T.; Ikuhara, Y.; Chiu, Y.-P.; Chang, C.-S.; Duan, C.-G.; He, Q.; Chu, Y.-H. A Metal-Insulator Transition of the Buried $\mathrm{MnO}_{2}$ Monolayer in Complex Oxide Heterostructure. Adv. Mater. 2016, 28, 9142-9151.

(27) Ohtomo, A.; Hwang, H. Y. A high-mobility electron gas at the $\mathrm{LaAlO}_{3} / \mathrm{SrTiO}_{3}$ heterointerface. Nature 2004, 427, 423-426.

(28) Liu, Z. Q.; Li, C. J.; Lü, W. M.; Huang, X. H.; Huang, Z.; Zeng, S. W.; Qiu, X. P.; Huang, L. S.; Annadi, A.; Chen, J. S.; Coey, J. M. D.; Venkatesan, T.; Ariando Origin of the Two-Dimensional Electron Gas at $\mathrm{LaAlO}_{3} / \mathrm{SrTiO}_{3}$ Interfaces: The Role of Oxygen Vacancies and Electronic Reconstruction. Phys. Rev. X 2013, 3, 021010.

(29) Bristowe, N. C.; Philippe, G.; Littlewood, P. B.; Emilio, A. The origin of two-dimensional electron gases at oxide interfaces: insights from theory. J Phys. Condens. Matter. 2014, 26, 143201.

(30) Lee, P. W.; Singh, V. N.; Guo, G. Y.; Liu, H. J.; Lin, J. C.; Chu, Y. H.; Chen, C. $\mathrm{H}$.; Chu, M. W. Hidden lattice instabilities as origin of the conductive interface between insulating $\mathrm{LaAlO}_{3}$ and $\mathrm{SrTiO}_{3}$. Nat. Commun. 2016, 7, 12773.

(31) Kawasaki, M.; Takahashi, K.; Maeda, T.; Tsuchiya, R.; Shinohara, M.; Ishiyama, O.; Yonezawa, T.; Yoshimoto, M.; Koinuma, H. Atomic Control of the $\mathrm{SrTiO}_{3}$ Crystal Surface. Science 1994, 266, 1540-1542.

(32) Rijnders, G.; Blank, D. H. A.; Choi, J.; Eom, C.-B. Enhanced surface diffusion through termination conversion during epitaxial $\mathrm{SrRuO}_{3}$ growth. Appl. Phys. Lett. 2004, 84, 505-507.

(33) Mott, N. F. Amorphous and Liquid Semiconductors Introductory talk; Conduction in non-crystalline materials. J. Non·Cryst. Solids 1972, 8, 1-18.

(34) Khondaker, S. I.; Shlimak, I. S.; Nicholls, J. T.; Pepper, M.; Ritchie, D. A. Two-dimensional hopping conductivity in a $\mathrm{GaAs} / \mathrm{Al}_{\mathrm{x}} \mathrm{Ga}_{1-\mathrm{x}} \mathrm{As}$ heterostructure. Phys. Rev. B 1999, 59, 4580-4583.

(35) Withers, F.; Russo, S.; Dubois, M.; Craciun, M. F. Tuning the electronic transport properties of graphene through functionalisation with fluorine. Nanoscale Res. Lett. 2011, 6, 1-11.

(36) Nakagawa, N.; Hwang, H. Y.; Muller, D. A. Why some interfaces cannot be sharp. Nat. Mater. 2006, 5, 204-209.

(37) Agrestini, S.; Hu, Z.; Kuo, C. Y.; Haverkort, M. W.; Ko, K. T.; Hollmann, N.; Liu, Q.; Pellegrin, E.; Valvidares, M.; Herrero-Martin, J.; Gargiani, P.; Gegenwart, P.; Schneider, M.; Esser, S.; Tanaka, A.; Komarek, A. C.; Tjeng, L. 
H. Electronic and spin states of $\mathrm{SrRuO}_{3}$ thin films: An x-ray magnetic circular dichroism study. Phys. Rev. B 2015, 91, 075127.

(38) Si, L.; Zhong, Z.; Tomczak, J. M.; Held, K. Route to room-temperature ferromagnetic ultrathin $\mathrm{SrRuO}_{3}$ films. Phys. Rev. B 2015, 92, 041108.

(39) Liu, H.-J.; Wei, T.-C.; Zhu, Y.-M.; Liu, R.-R.; Tzeng, W.-Y.; Tsai, C.-Y.; Zhan, Q.; Luo, C.-W.; Yu, P.; He, J.-H.; Chu, Y.-H.; He, Q. Strain-Mediated Inverse Photoresistivity in $\mathrm{SrRuO}_{3} / \mathrm{La}_{0.7} \mathrm{Sr}_{0.3} \mathrm{MnO}_{3}$ Superlattices. Adv. Funct. Mater. 2016, 26, 729-737.

(40) Wei, T.-C.; Wang, H.-P.; Liu, H.-J.; Tsai, D.-S.; Ke, J.-J.; Wu, C.-L.; Yin, Y.-P.; Zhan, Q.; Lin, G.-R.; Chu, Y.-H.; He, J.-H. Photostriction of strontium ruthenate. Nat. Commun. 2017, 8, 15018.

(41) Huang, B.-C.; Chiu, Y.-P.; Huang, P.-C.; Wang, W.-C.; Tra, V. T.; Yang, J.-C.; He, Q.; Lin, J.-Y.; Chang, C.-S.; Chu, Y.-H. Mapping Band Alignment across Complex Oxide Heterointerfaces. Phys. Rev. Lett. 2012, 109, 246807.

(42) Feenstra, R. M. Tunneling spectroscopy of the (110) surface of direct-gap III-V semiconductors. Phys. Rev. B 1994, 50, 4561-4570.

(43) Ariando; Wang, X.; Baskaran, G.; Liu, Z. Q.; Huijben, J.; Yi, J. B.; Annadi, A.; Barman, A. R.; Rusydi, A.; Dhar, S.; Feng, Y. P.; Ding, J.; Hilgenkamp, H.; Venkatesan, T. Electronic phase separation at the $\mathrm{LaAlO}_{3} / \mathrm{SrTiO}_{3}$ interface. Nat. Commun. 2011, 2, 188.

(44) Kresse, G.; Hafner, J. Ab initio molecular dynamics for liquid metals. Phys. Rev. $B$ 1993, 47, 558-561.

(45) Kresse, G.; Hafner, J. Ab initio molecular-dynamics simulation of the liquid-metal-amorphous-semiconductor transition in germanium. Phys. Rev. B 1994, 49, 14251-14269.

(46) Gupta, K.; Mandal, B.; Mahadevan, P. Strain-induced metal-insulator transition in ultrathin films of $\mathrm{SrRuO}_{3}$. Phys. Rev. B 2014, 90, 125109. 


\section{Figures}

(a)

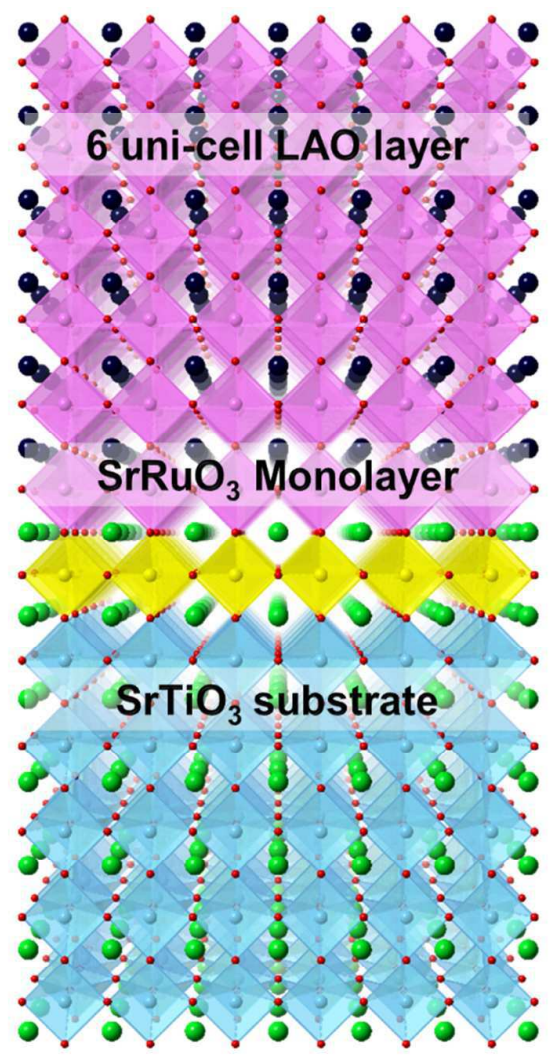

(b)

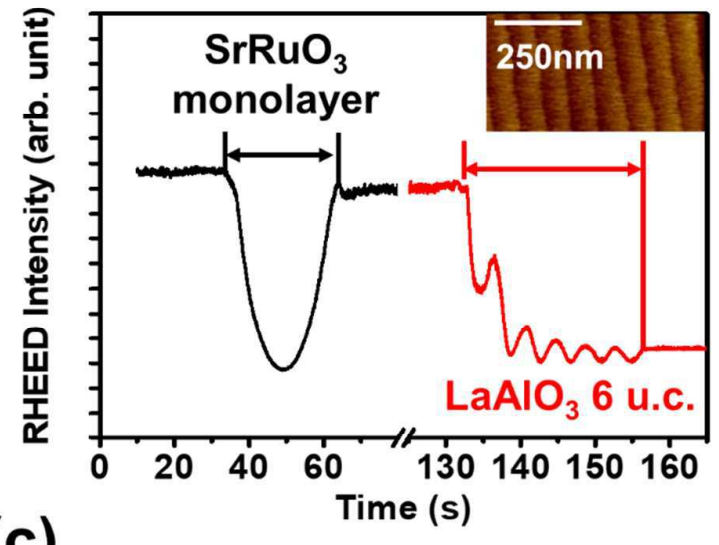

(c)

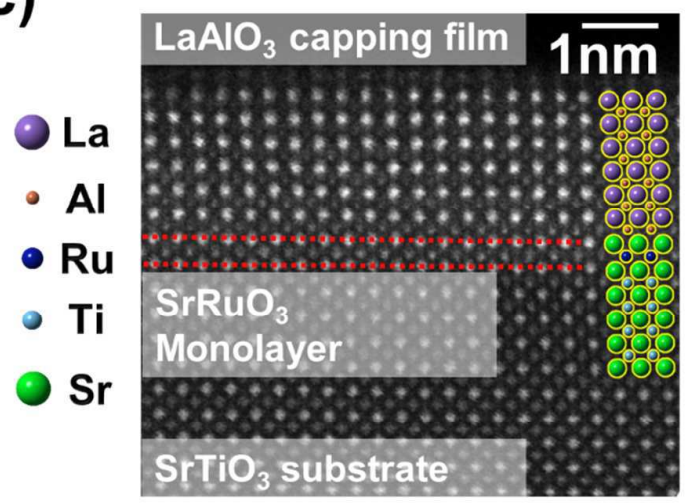

Figure 1. (a). The schematic of the SRO monolayer capped with the 6-unit-cell LAO layer grown on the $\mathrm{TiO}_{2}$ terminated STO substrates. (b) Real-time monitoring of RHEED intensity for the SRO monolayer and LAO capping layer. The inset in (b) shows the typical morphology of atomically flat surface. (c) The SRO monolayer sample identified by the HAADF-STEM measurement. 


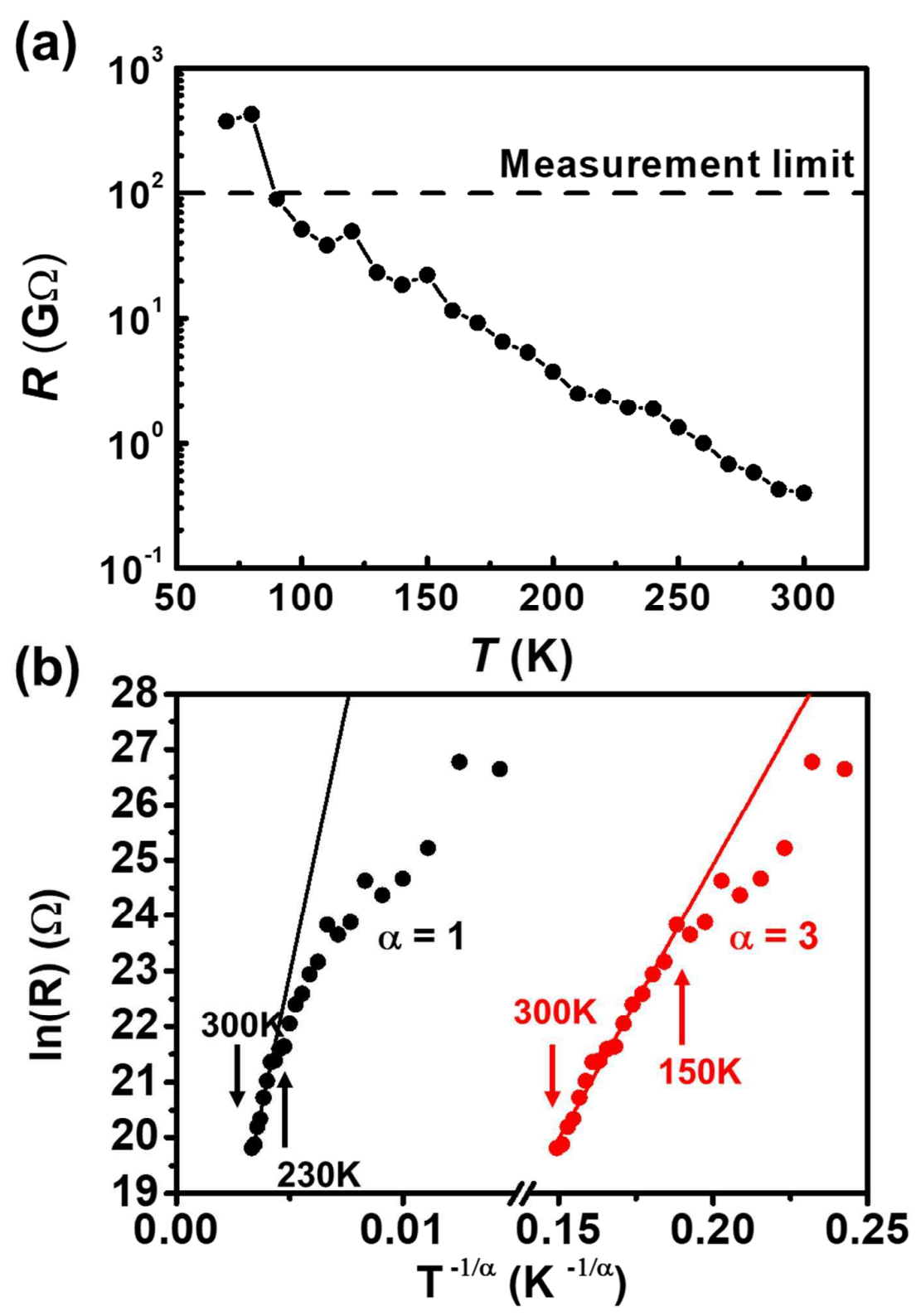

Figure 2. (a) A semiconducting behavior of the SRO monolayer can be observed by capped with the LAO thin layer. (b) The plot of $\ln (R(T))$ versus $(1 / T)^{1 / \alpha}$, where $\alpha=1$ is the fitting result from Arrhenius equation, and $\alpha=3$ is according to the model of 2D Mott VRH. The wider fitted range in the latter model supports that 2D transport behavior for this SRO monolayer. 
(a)

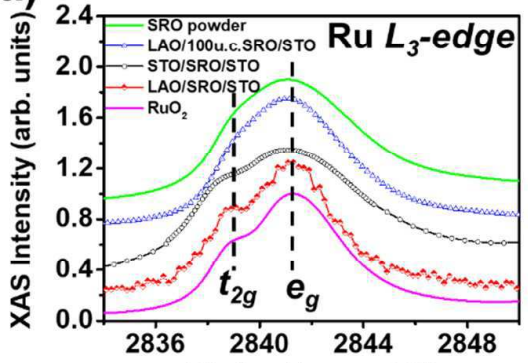

(b)

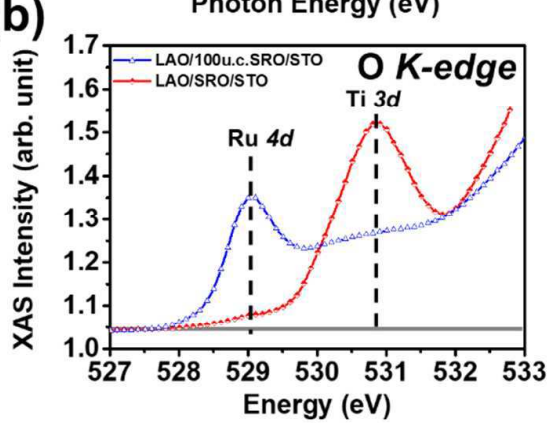

(c)
More stressed
Less stressed SRO monolayer SRO monolayer
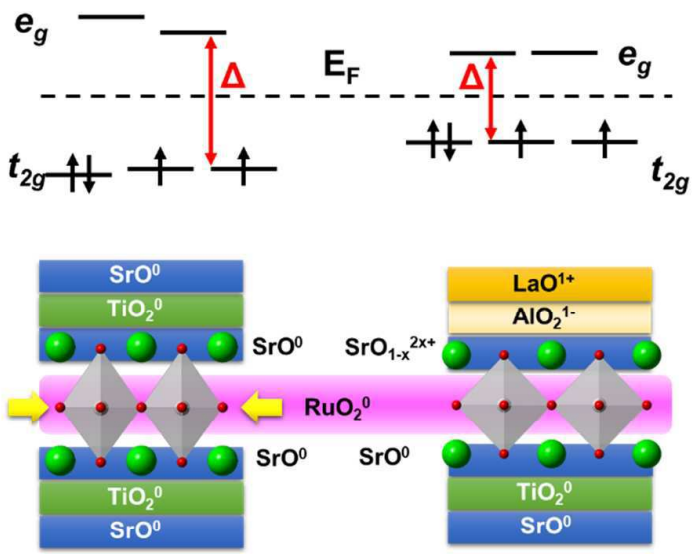

Figure 3. (a) The XAS spectra across the Ru $L$-edges taken from the SRO monolayers with different capping layers, and the control samples of the 100-unit-cell SRO with the LAO capping layer, the standard SRO and $\mathrm{RuO}_{2}$ powders. No obvious peak shift on the signal of $e_{g}$ and $t_{2 g}$ orbitals describes no valence change of $\mathrm{Ru}$ ions in all samples. Higher intensity ratio of $e_{g} / t_{2 g}$ also indicates less distortion of $\mathrm{Ru}$ octahedrons. (b) The XAS spectra across $\mathrm{O} K$-edge reveal the $\mathrm{Ru} 4 d-\mathrm{O} 2 p(\sim 529.04$ $\mathrm{eV})$ and $\mathrm{Ti} 3 d-\mathrm{O} 2 p(\sim 530.86 \mathrm{eV})$ hybridizations. The nearly unchanged peak positions between the SRO monolayer and the bulk SRO film suggests that the top SrO plane of SRO can effectively avoid oxygen deficiency at the $\mathrm{RuO}_{2}$ plane and the neighboring $\mathrm{TiO}_{2}$ planes inside the substrates. (c) The model of band gap modulation in the SRO monolayer due to the crystal field splitting by different capping layers. 

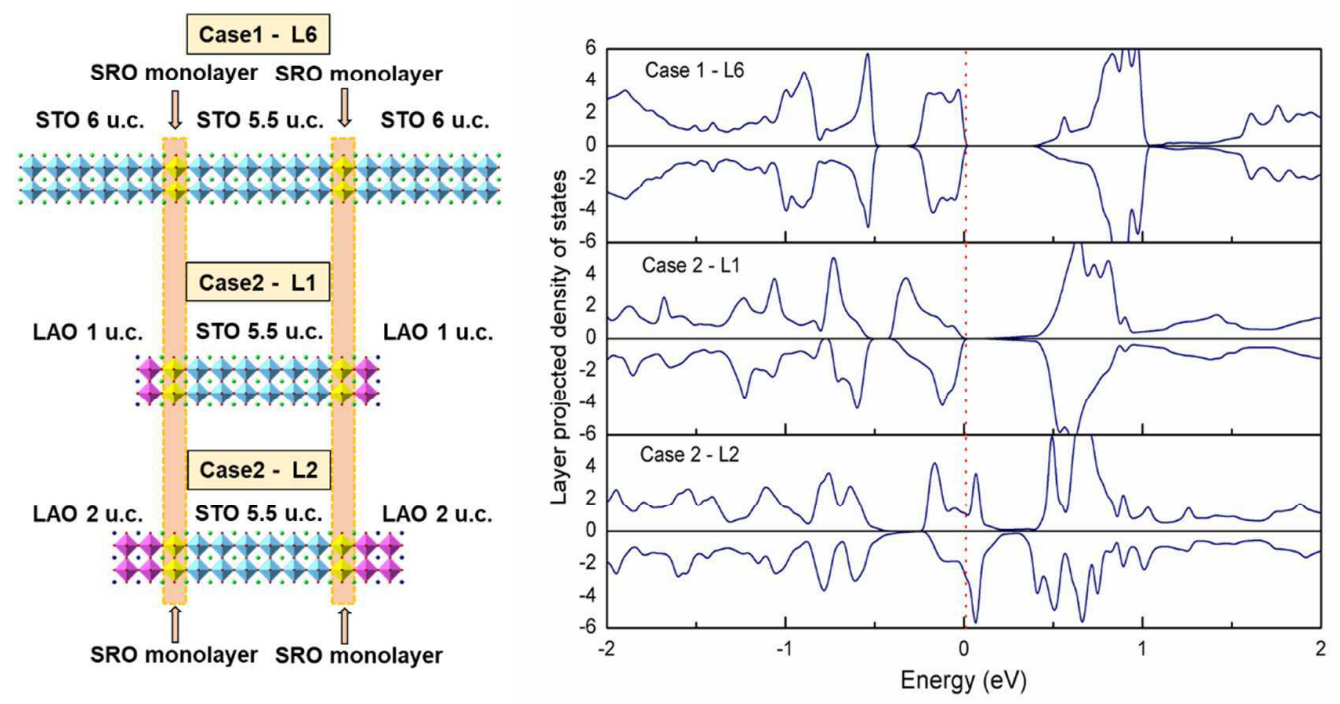

Figure 4. The slab models of case $1 \quad-\quad$ L6 $\left((\mathrm{STO})_{6}-(\mathrm{SRO})_{1.5}-(\mathrm{STO})_{5.5}-(\mathrm{SRO})_{1.5}-(\mathrm{STO})_{6}\right), \quad$ case $\quad 2 \quad-\quad$ L1 $\left((\mathrm{LAO})_{1}-(\mathrm{SRO})_{1.5}-(\mathrm{STO})_{5.5}-(\mathrm{SRO})_{1.5}-(\mathrm{LAO})_{1}\right), \quad$ and $\quad$ case $2 \quad-\quad \mathrm{L} 2$ $\left((\mathrm{LAO})_{2}-(\mathrm{SRO})_{1.5}-(\mathrm{STO})_{5.5}-(\mathrm{SRO})_{1.5}-(\mathrm{LAO})_{2}\right)$ for calculation and the respective layer projected density of states of these SRO monolayers. The Fermi level is given by the dashed line. 
(a)

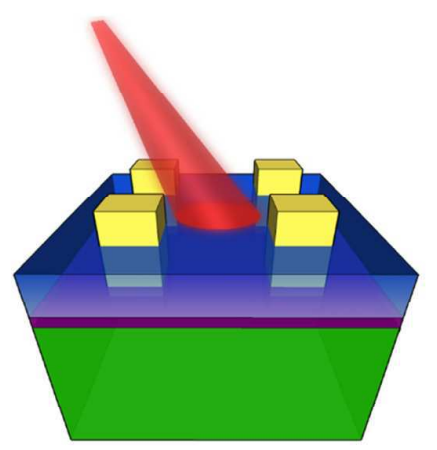

(c)

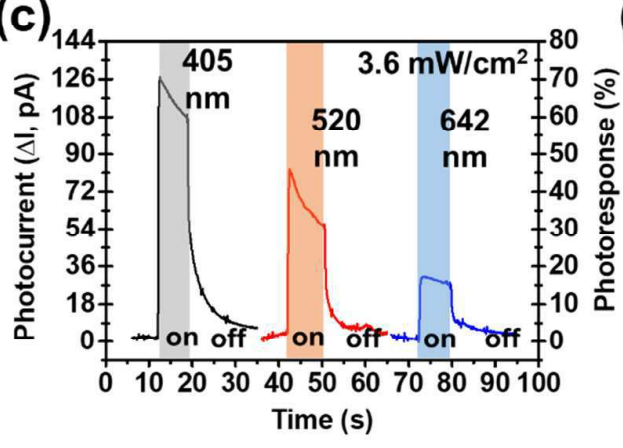

(b)

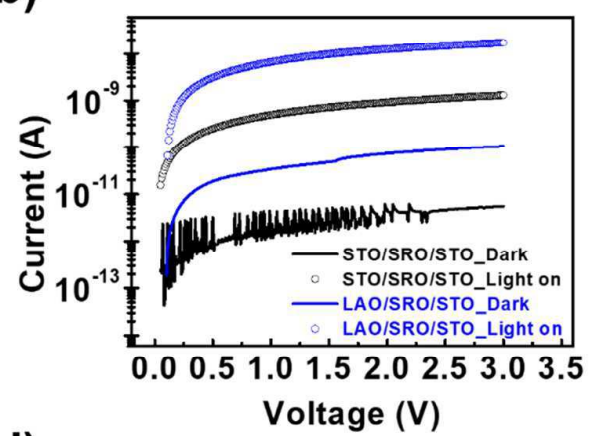

(d)

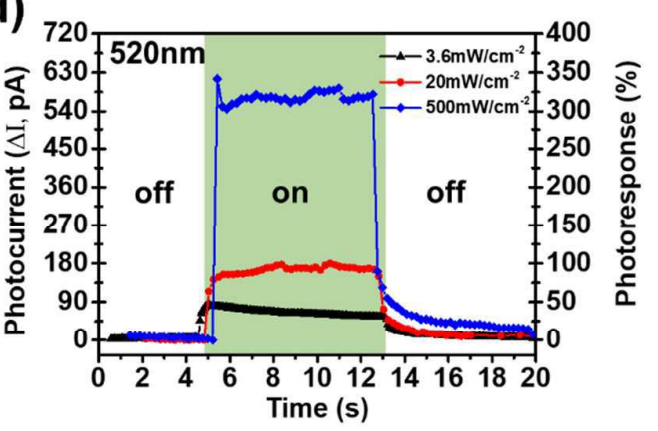

Figure 5. (a) The schematic of the photoresponse measurement. (b) The I-V curves for $\mathrm{LAO} / \mathrm{SRO} / \mathrm{STO}$ and $\mathrm{STO} / \mathrm{SRO} / \mathrm{STO}$ samples under the photon source of $350 \mathrm{~nm}$ with power density of $0.5 \mathrm{~mW} / \mathrm{cm}^{2}$. (c) The photocurrent and photoresponse of the SRO monolayer at three photon sources with $405 \mathrm{~nm}, 520 \mathrm{~nm}$, and $642 \mathrm{~nm}$. (D) The power dependent photocurrent and photoresponse of the SRO monolayer performed at $520 \mathrm{~nm}$, where the variation of photocurrent to dark current can be as high as $325 \%$. 


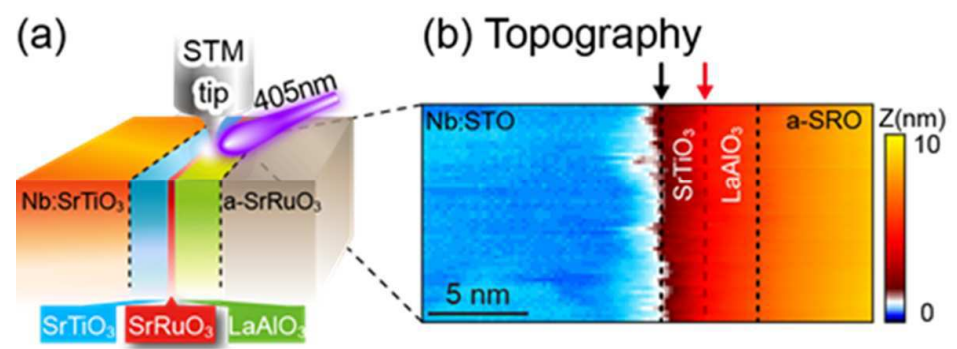

In dark

(c) dl/dV image $\mathrm{SrRuO}_{3}$

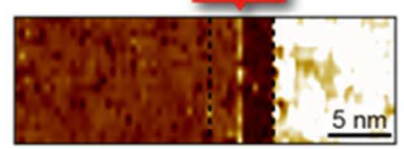

(e) Band alignment
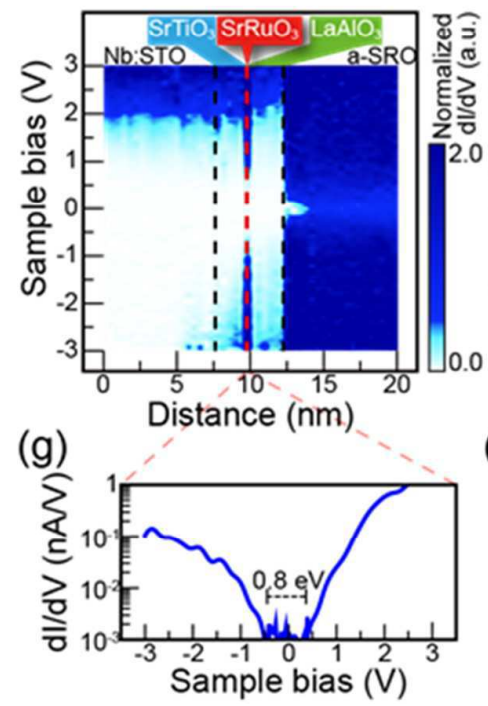

(d) $d l / d V$ image

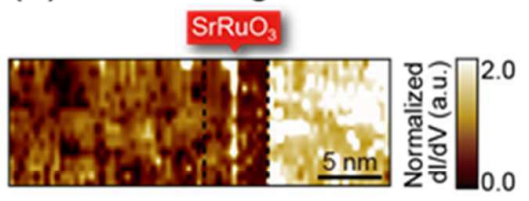

(f) Band alignment

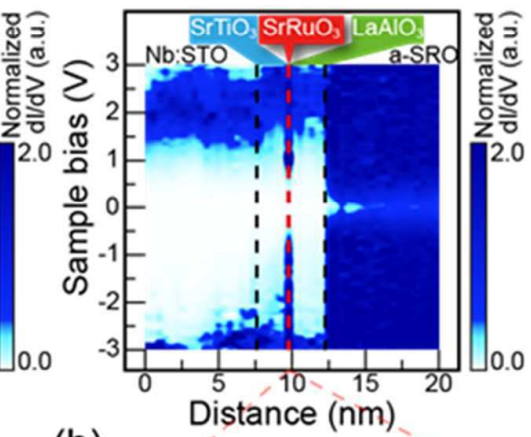

(h)

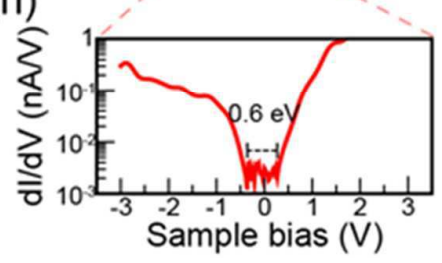

Figure 6. (a) Schematic diagram of cross-sectional STM used to measure the photo-modulated electronic properties of the SRO monolayer. (b) Typical cross-sectional topography image of the SRO monolayer at sample bias of $+2.0 \mathrm{~V}$. (c) and (d) are STS spectroscopic dI/dV images of the SRO monolayer at sample bias of $+2.0 \mathrm{~V}$ in dark and under illumination, respectively. (e) and (f) are band alignments and corresponding atomic-scale resolutions of electronic properties across the SRO 


\author{
monolayer extracted from (c) and (d), respectively. Further analysis of the energy gap \\ from $\mathrm{dI} / \mathrm{dV}$ curves of the SRO monolayer in dark and under illumination can be \\ observed in $(\mathrm{g})$ and $(\mathrm{h})$, respectively.
}


(a) (b)

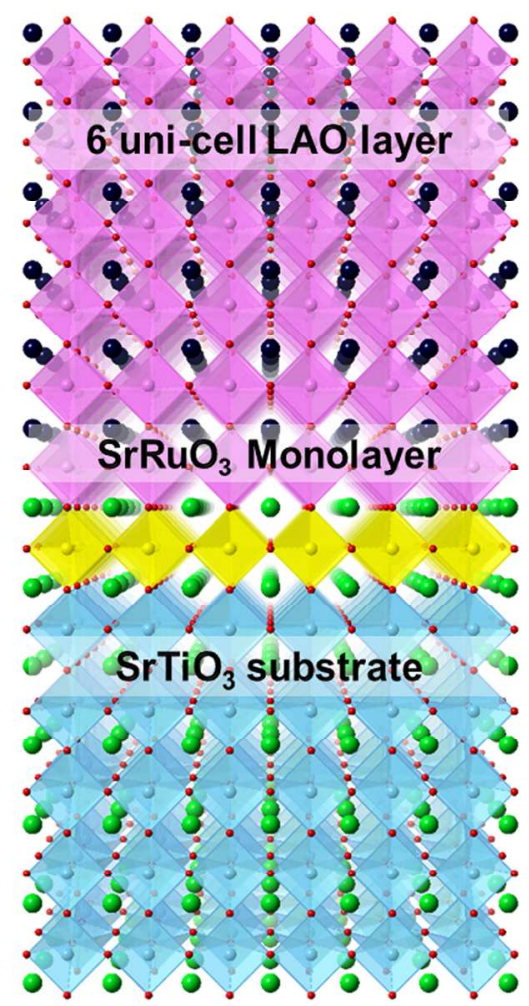

(c)
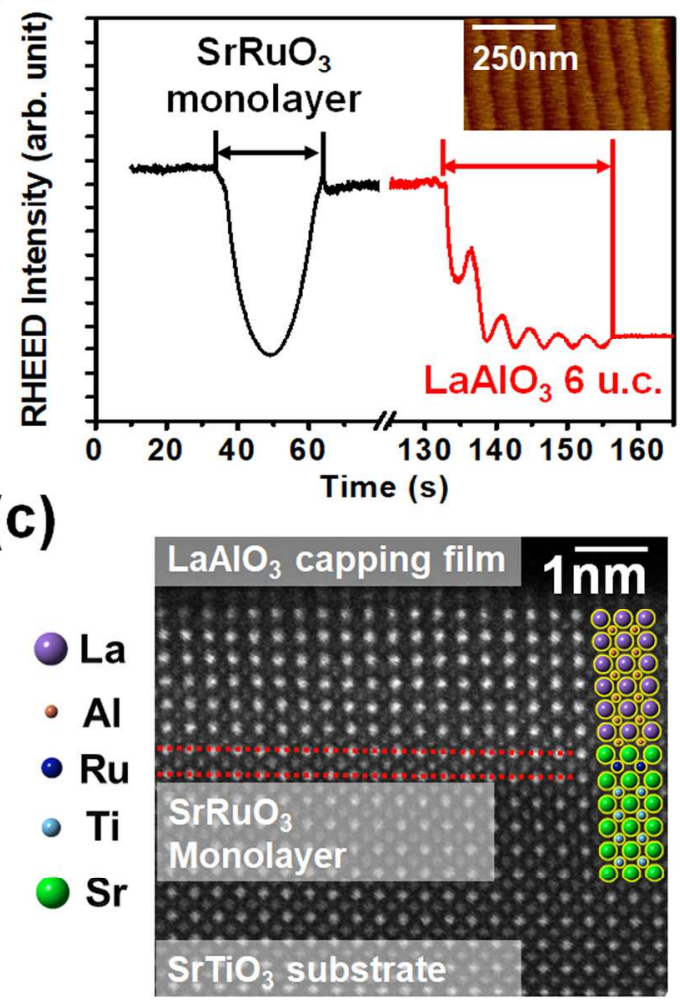

Figure 1. (a). The schematic of the SRO monolayer capped with the 6-unit-cell LAO layer grown on the $\mathrm{TiO}_{2}$ terminated STO substrates. (b) Real-time monitoring of RHEED intensity for the SRO monolayer and LAO capping layer. The inset in (b) shows the typical morphology of atomically flat surface. (c) The SRO monolayer sample identified by the HAADF-STEM measurement.

$161 \times 133 \mathrm{~mm}(300 \times 300 \mathrm{DPI})$ 


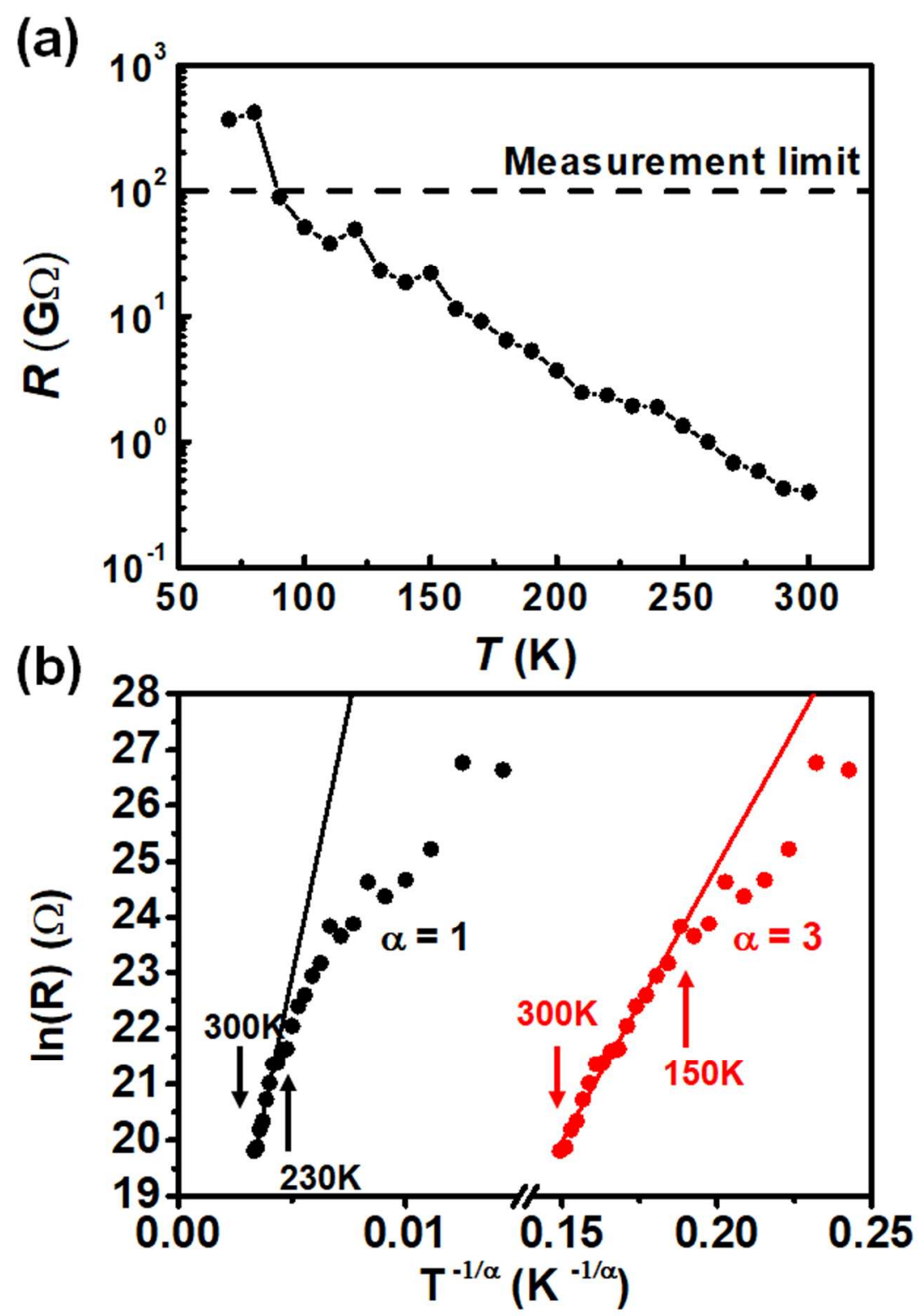

Figure 2. (a) A semiconducting behavior of the SRO monolayer can be observed by capped with the LAO thin layer. (b) The plot of $\ln (R(T))$ versus, where $\mathrm{a}=1$ is the fitting result from Arrhenius equation, and $\mathrm{a}=3$ is according to the model of 2D Mott VRH. The wider fitted range in the latter model supports that 2D transport behavior for this SRO monolayer. 
(a)
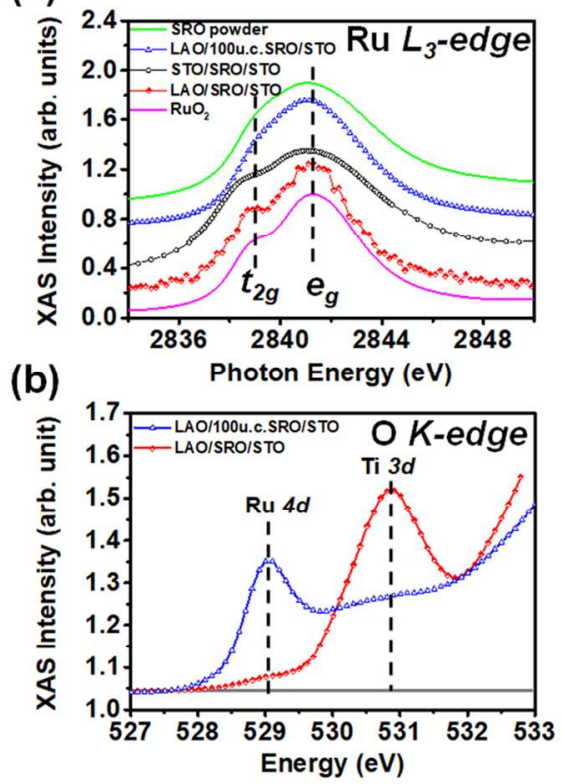

(c)
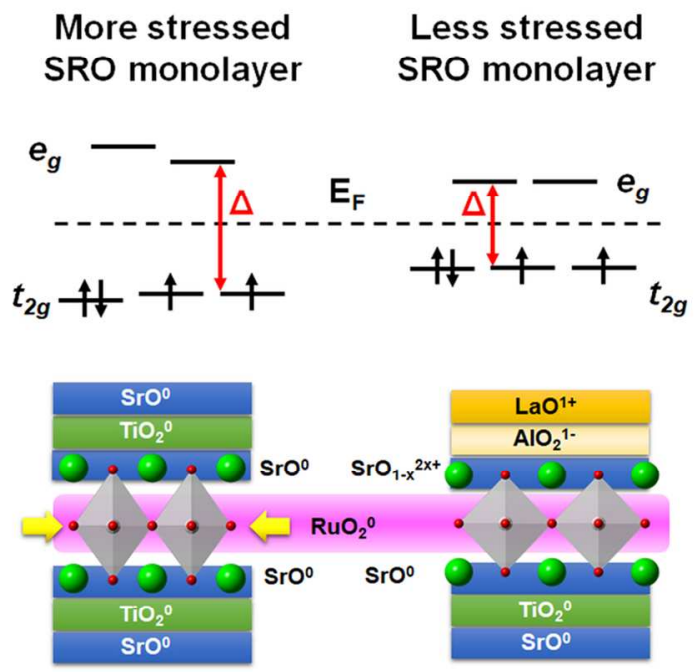

Figure 3. (a) The XAS spectra across the Ru L-edges taken from the SRO monolayers with different capping layers, and the control samples of the 100-unit-cell SRO with the LAO capping layer, the standard SRO and $\mathrm{RuO}_{2}$ powders. No obvious peak shift on the signal of $e_{g}$ and $t_{2 g}$ orbitals describes no valence change of Ru ions in all samples. Higher intensity ratio of $e_{g} / t_{2 g}$ also indicates less distortion of Ru octahedrons. (b) The

XAS spectra across O $K$-edge reveal the Ru $4 d-\mathrm{O} 2 p(\sim 529.04 \mathrm{eV})$ and Ti $3 d-\mathrm{O} 2 p(\sim 530.86 \mathrm{eV})$ hybridizations. The nearly unchanged peak positions between the SRO monolayer and the bulk SRO film suggests that the top SrO plane of SRO can effectively avoid oxygen deficiency at the $\mathrm{RuO}_{2}$ plane and the neighboring $\mathrm{TiO}_{2}$ planes inside the substrates. (c) The model of band gap modulation in the SRO monolayer due to the crystal field splitting by different capping layers.

$112 \times 71 \mathrm{~mm}(300 \times 300$ DPI $)$ 


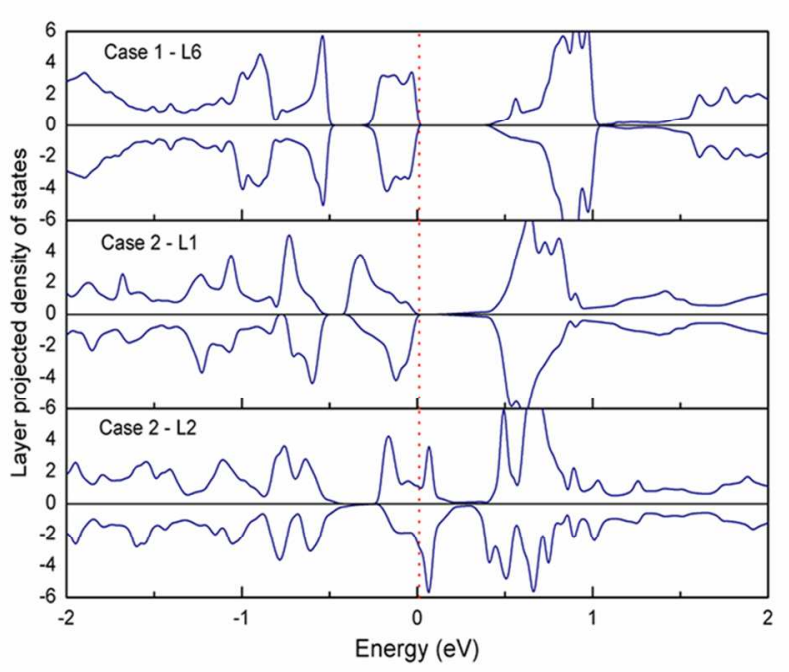

Figure 4. The slab models of case 1 - L6 ((STO)6-(SRO) $\left.)_{1.5}-(\mathrm{STO})_{5.5}-(\mathrm{SRO})_{1.5}-(\mathrm{STO})_{6}\right)_{\text {, }}$ case 2 - L1 ((LAO) - $^{-}$ $\left.(\mathrm{SRO})_{1.5}-(\mathrm{STO})_{5.5}-(\mathrm{SRO})_{1.5}-(\mathrm{LAO})_{1}\right)_{\text {, }}$ and case $2-\mathrm{L}_{2}\left((\mathrm{LAO})_{2}-(\mathrm{SRO})_{1.5}-(\mathrm{STO})_{5.5}-(\mathrm{SRO})_{1.5}-(\mathrm{LAO})_{2}\right)$ for calculation and the respective layer projected density of states of these SRO monolayers. The Fermi level is given by the dashed line.

$94 \times 50 \mathrm{~mm}(300 \times 300 \mathrm{DPI})$ 
(a)

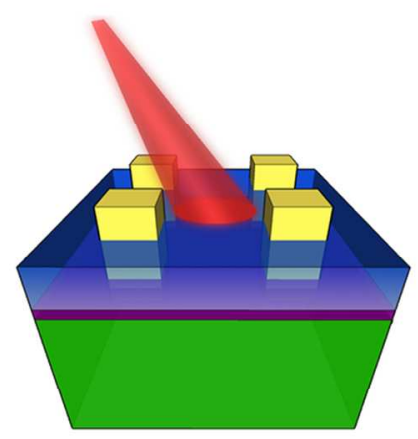

(c)

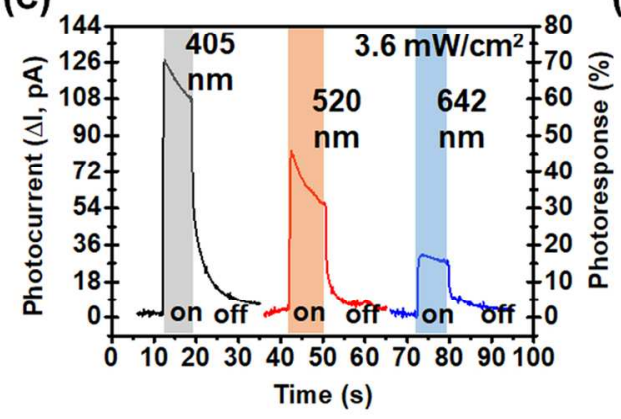

(b)

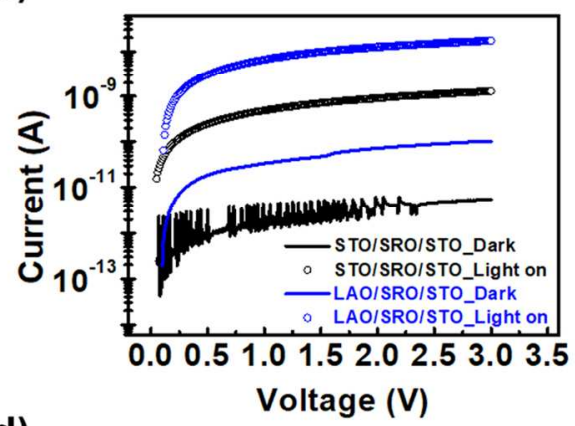

(d)

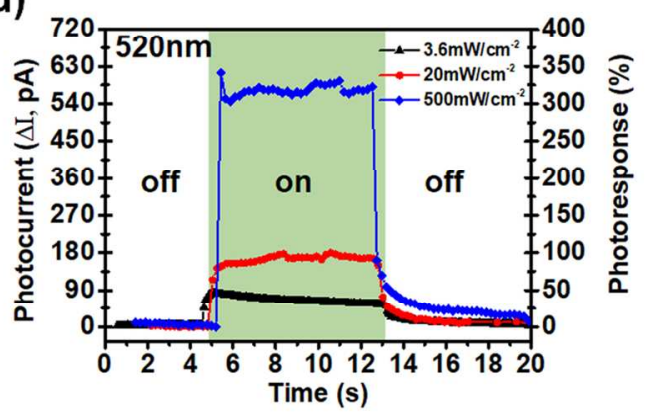

Figure 5. (a) The schematic of the photoresponse measurement. (b) The I-V curves for LAO/SRO/STO and STO/SRO/STO samples under the photon source of $350 \mathrm{~nm}$ with power density of $0.5 \mathrm{~mW} / \mathrm{cm}^{2}$. (c) The photocurrent and photoresponse of the SRO monolayer at three photon sources with $405 \mathrm{~nm}, 520 \mathrm{~nm}$, and $642 \mathrm{~nm}$. (D) The power dependent photocurrent and photoresponse of the SRO monolayer performed at 520 $\mathrm{nm}$, where the variation of photocurrent to dark current can be as high as $325 \%$.

$127 \times 90 \mathrm{~mm}(300 \times 300 \mathrm{DPI})$ 


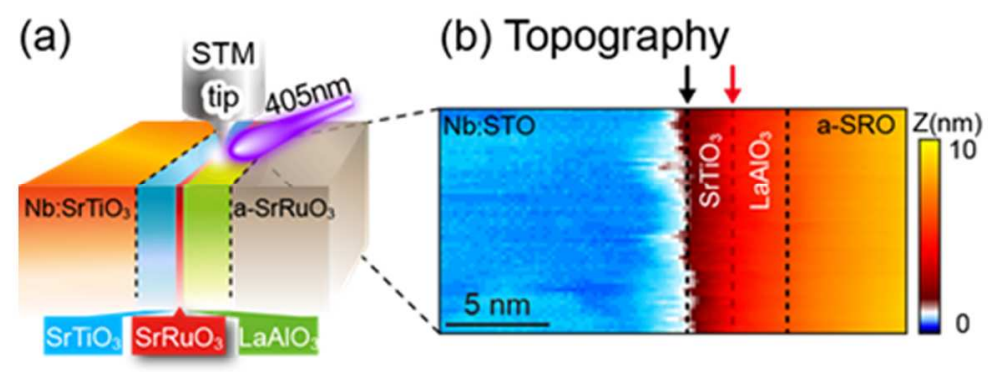

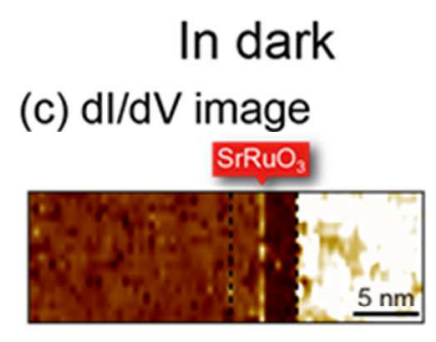

(e) Band alignment
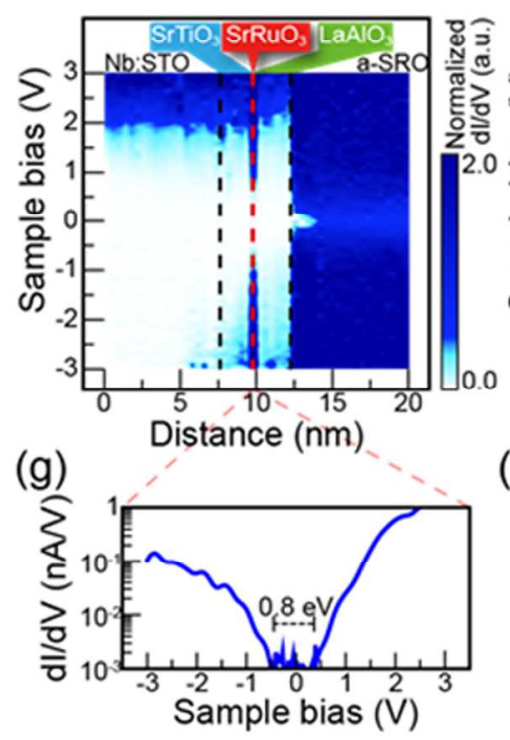

(d) $d l / d V$ image

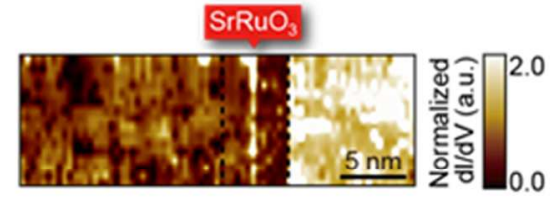

(f) Band alignment

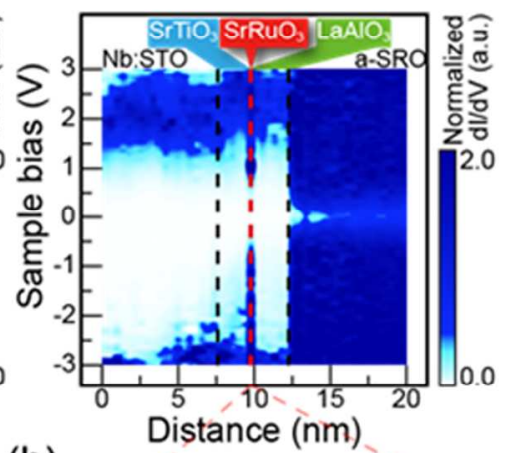

(h)

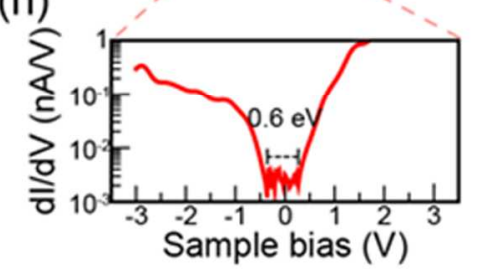

Figure 6. (a) Schematic diagram of cross-sectional STM used to measure the photo-modulated electronic properties of the SRO monolayer. (b) Typical cross-sectional topography image of the SRO monolayer at sample bias of $+2.0 \mathrm{~V}$. (c) and (d) are STS spectroscopic dI/dV images of the SRO monolayer at sample bias of $+2.0 \mathrm{~V}$ in dark and under illumination, respectively. (e) and ( $f$ ) are band alignments and corresponding

atomic-scale resolutions of electronic properties across the SRO monolayer extracted from (c) and (d), respectively. Further analysis of the energy gap from dI/dV curves of the SRO monolayer in dark and under illumination can be observed in $(g)$ and $(h)$, respectively.

$$
232 \times 351 \mathrm{~mm}(300 \times 300 \text { DPI })
$$




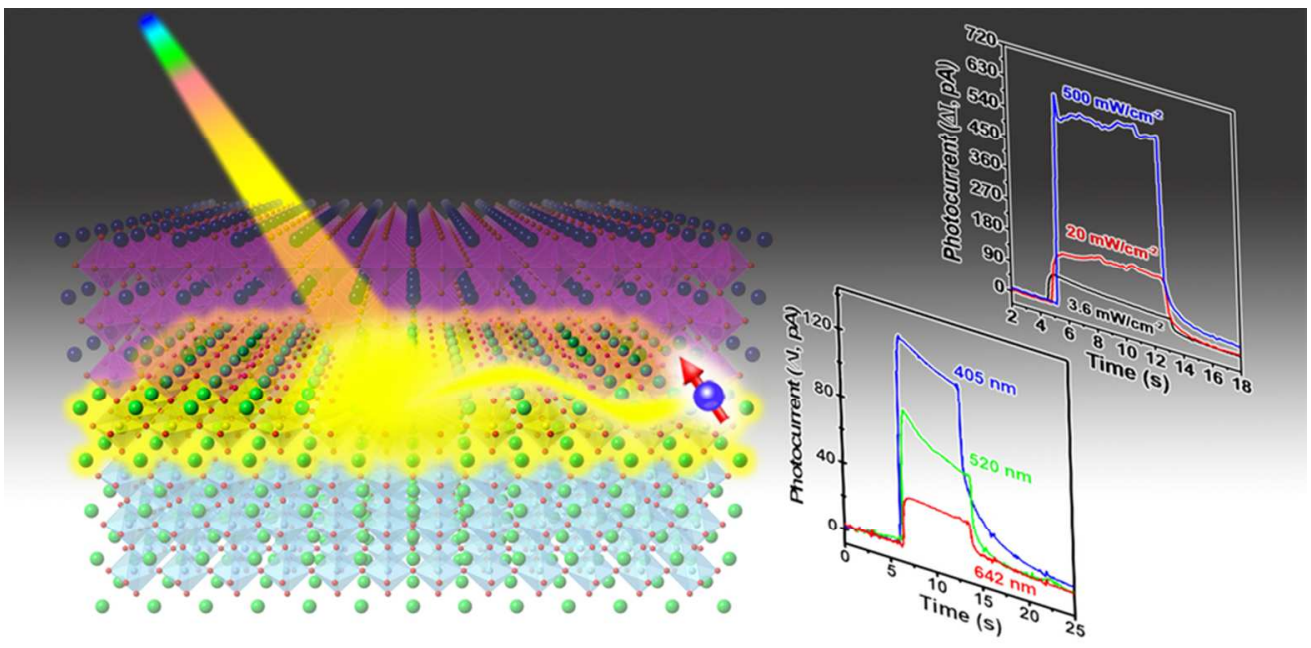

$80 \times 40 \mathrm{~mm}(300 \times 300$ DPI $)$ 